\title{
Different Models of a Scaled Experimental Running Gear for the DLR RailwayDynamics Library
}

\author{
Christoph Schwarz Andreas Heckmann Alexander Keck \\ Institute of System Dynamics and Control, German Aerospace Center (DLR), 82234 Wessling \\ \{Christoph.Schwarz, Andreas.Heckmann, Alexander.Keck\}@dlr.de
}

\begin{abstract}
The DLR internal project "Next Generation Train" (NGT) deals with a high-speed train in a double-deck configuration. To realize the two continuous floors, a single wheel running gear configuration is selected. Equipped with independently rotating wheels instead of a usual wheel-set, a track guidance control becomes necessary. In terms of an advanced control and observer development the implementation of validated simulation models is absolutely essential. Therefore, the paper gives a short overview of the hardware of the scaled Experimental Running Gear on the DLR roller rig representing the NGT single wheel running gear. Using the DLR RailwayDynamics Library three different models of the running gear are implemented, which vary in complexity and can be used for different analysis methods. Finally, some significant simulation results of the particular simulation models are presented and discussed.

Keywords: railway vehicle dynamics, running gear, analytical modeling
\end{abstract}

\section{Introduction}

In 1985 a roller rig was established at DLR. Since then various aspects of the railway vehicle dynamics have been investigated using constantly advanced running gears. The current configuration represents the single axle running gear of the DLR internal project "Next Generation Train" (NGT) (Winter et al., 2011). This project is targeted on a high-speed train in lightweight design with high demands for energy efficiency and the passenger capacity. To achieve these goals the train is designed in a double-deck configuration with continuous floors on both levels. Therefore, the running gear is equipped with independently rotating wheels (IRW) mounted on an axle bridge and individually driven. To stabilize the running gear dynamics, which are unstable at higher speeds (Wickens, 2003), a mechatronic track guidance is applied to ensure the safe service of the train and to reduce the wheel and rail wear.

Regarding the synthesis of a model based control and an extensive analysis of the running gear system, the development of an appropriate and validated but at the same time simple simulation model is essential. In a first step, the hardware of the scaled running gear on the DLR roller rig is described in Section 2. Furthermore, the paper establishes three different simulation models in Section 3 varying in the level of complexity and implemented using the DLR RailwayDynamics Library. Some simulation results as well as a comparison thereof are delineated in Section 4. Finally, in Section 5 an outlook to the future work is given together with conclusions.

\section{Hardware of the running gear on the roller rig}

The actual running gear operating on the roller rig is a scaled $1: 5$ version of the single axle running gear conceived for the intermediate wagons of the NGT. The major components of the Experimental Running Gear are the central frame, the two axle bridges, and the four wheels. Figure 1 illustrates the particular modules as well as the mechanical degrees of freedom relevant for the mechatronic track guidance control. Using the indices $i=f, r$ (front, rear axle bridge) and $j=r, l$ (right, left wheel), respectively, the DOFs are the lateral displacements $y_{f}$ and $y_{r}$ of the center point of the axle bridge $P$ with respect to the railroad centerline, the yaw

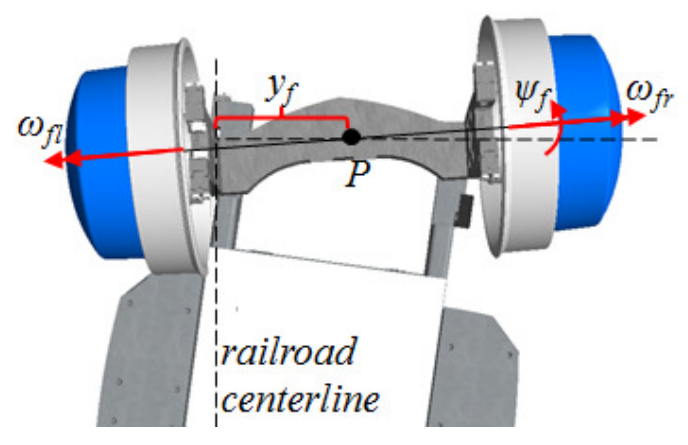

Figure 1. Half model of the running gear with mechanical degrees of freedom. 


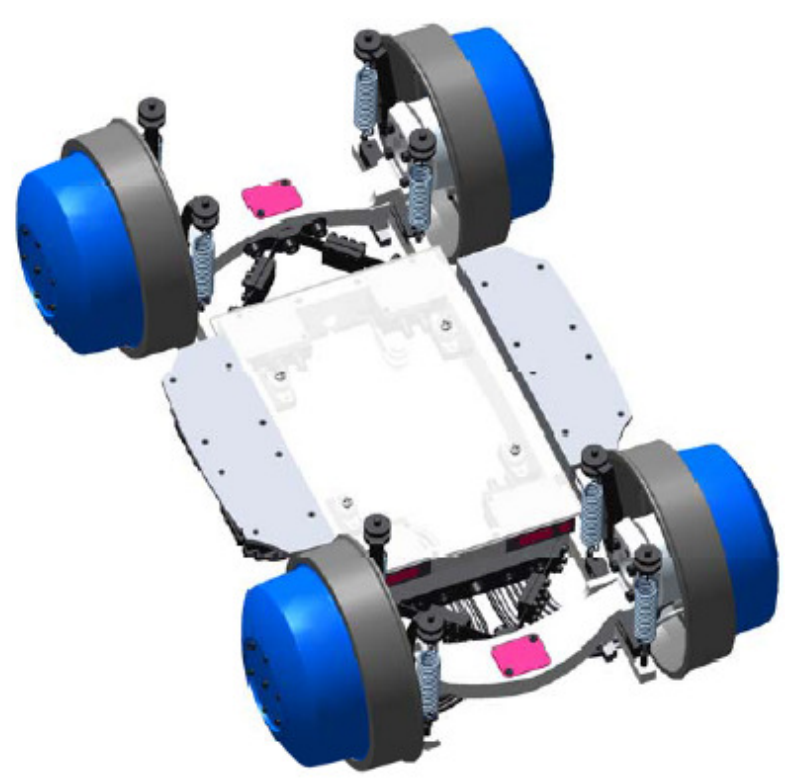

Figure 2. Detailed CAD model of the current running gear design.

angle of each axle bridge $\psi_{f}$ and $\psi_{r}$ and the four angular wheel velocities $\omega_{f r}, \omega_{f l}, \omega_{r r}$ and $\omega_{r l}$.

The central frame is a screwed construction and very small manufacturing tolerances are demanded on the particular parts. In addition, the frame is mounted to the roller rig by a lemniscate guidance, that blocks the longitudinal motion of the running gear but allows for lateral and vertical motions as well as yawing. Laser sensors used for the measurements of $y_{i}$ and $\psi_{i}$ are attached to the frame just as the converters of the motors. Furthermore, each axle bridge is interconnected to the frame by two leaf springs as it can be seen in Figure 2. The springs act on the one hand as an axle bridge guidance with respect to the frame and on the other hand as vertical suspension. The adjustment of these springs in a V-shape guarantees a guidance free of clearance and allows radial steering of the axle bridges, what is necessary for the mechatronic track guidance. This leaf spring guidance concept and an improved cable guidance from the laser sensors and converters to the target PC are the outcome of the latest redesign of the running gear in 2014. Another enhancement of the newly constructed running gear are the low torsional stiffness of the frame and the additional vertical springs, that can be added in order to adapt for an optional, additional load. The vertical springs and the leaf springs lead to a rotational yaw stiffness between the axle bridges and the central frame, which has to be taken into account in the development of the track guidance control.

The IRWs are individually driven by permanentmagnet synchronous motors. In contrast to the real NGT setup, these in-wheel drives only have to deliver the control torque but not the traction torque, which is generated by the rollers. There are two independent control torques, one for the leading and one for the trailing axle bridge, since the torques of the right and the left motor

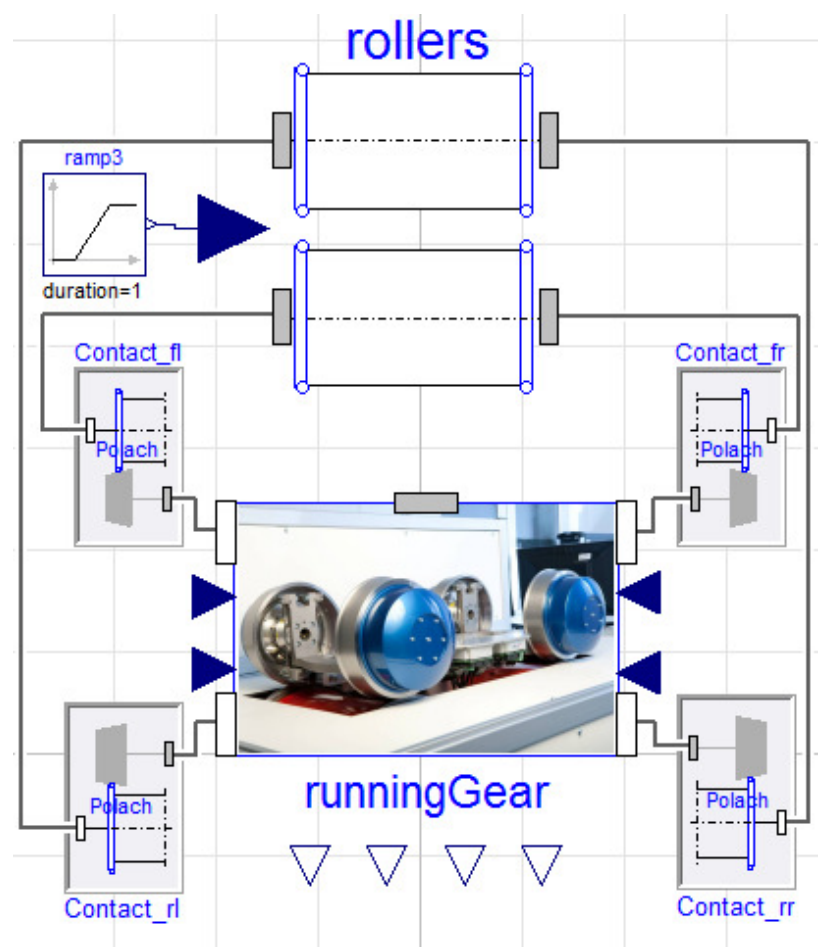

Figure 3. Modelica model of the running gear on the roller rig.

are equal in amount but opposite in direction. The two torques are calculated by separate, but identically parameterized controllers. The cascaded control structure implies an inner PD loop for the yaw angle and an outer PI loop for the lateral displacement.

\section{Modeling aspects of the scaled run- ning gear}

Before describing the specific models with their characteristics some general aspects are pointed out that all three of them have in common. Firstly, the limitation of the actuator torque representing a non-linearity is modeled as part of the controller. Furthermore, the angular wheel velocities $\omega_{i j}$ are negative, since the angular roller velocity $\omega_{R}=\frac{v_{R}}{r_{R}}$ is positive in case of a positive $v_{R}$. Another common aspect of the models described hereafter is to idealize the wheel profile as perfectly conical with the cone angle $d$.

\subsection{Detailed multibody Model}

The multibody model of the running gear is divided into two parts: the running gear itself and the contact models of each wheel-rail pair, see Figure 3. According to (Heckmann et al., 2014) there are two options for the contact model: Kalker's linear theory and the theory formulated by Polach. Due to the more accurate contact formulation the latter theory is used to calculate the creep forces $\boldsymbol{f}=\left(f_{x}, f_{y}, l_{z}\right)^{T}$, with the longitudinal creep 


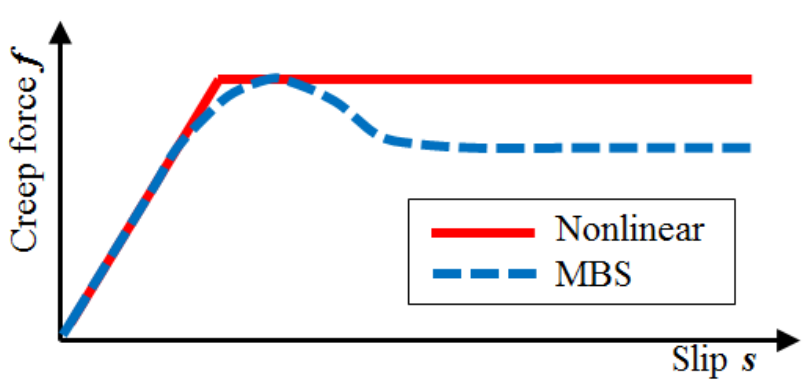

Figure 4. Creep forces in relation to the spin for the MBS and the nonlinear model(Knothe and Stichel, 2003).

force $f_{x}$, the lateral creep force $f_{y}$ and the torque $l_{z}$. The nonlinear relation between the creep forces and the slip $\boldsymbol{s}=\left(s_{x}, s_{y}, \phi_{z}\right)^{T}$, with the longitudinal slip $s_{x}$, the lateral slip $s_{y}$ and the spin $\phi_{z}$, is illustrated in Figure 4. In addition, the shear modulus $G$ and the Kalker coefficients $C_{11}, C_{22}, C_{23}$ and $C_{33}$ determine the calculation of $f$. The Kalker coefficients depend on the semi-axes of the contact ellipse $a$ and $b$ and are stored in look-up tables in the contact modules.

The running gear model comprises all of the major components described in the previous section and is in turn divided into different substructures. On the top level the interfaces for the in- and outputs $\tau_{i} j, y_{i}$ and $\psi_{i}$ are implemented, see Figure 3. Further parameters defined in this level are the wheel base $e$, the stiffness and the damping coefficient of the vertical spring/damper component as well as the body parameters of the central frame like its mass $M$ and the moments of inertia. In a first sublevel the DOFs of the central frame are modeled using three rotational and two translational joints that are connected in series. The leading and the trailing axle bridges are represented by identical substructures. Considering the axle bridge structure the rotational stiffness $k$ between the central frame and the axle bridge is modeled independently of the vertical spring. This component is positioned at the yaw joint of the axle bridge followed by the roll joint and the translational joint for the vertical displacement. Furthermore, the wheel gauge $f$, the nominal rolling radius of the wheels $r_{0}$ and the body parameters of the wheels and the axle bridge are defined. Another feature implemented in the axle bridge model is the transfer behavior from the requested controller torque to the actual angular wheel acceleration.

\subsection{Nonlinear analytical Model}

Since the overall aim is to generate a model that can be used for the development of a feed-forward as well as a feed-back control, another more simple model is necessary than the MBS model. Therefore, the complexity and the computational effort of the nonlinear model is reduced by carrying out some simplifications. First of all, the three rotational degrees of freedom of the central frame are locked, since for the true scale NGT rail- way car with $e=14 \mathrm{~m}$ and $f=1435 \mathrm{~mm}$ the influences of these rotations might be diminutive anyway. Though, for a later application to the true scale NGT the influences of the mass and the inertias of the car body have to be investigated. Another simplified aspect is the neglect of the vertical stiffness between the axle bridge and the frame, so the vertical displacement of the central frame is $z_{F}=\frac{z_{f}+z_{r}}{2}$. In addition, the width of the continuous roller rails is considered to be infinitesimal regarding the calculation of the position of the contact patch on the wheel surface. Furthermore, Kalker's linear theory (Kalker, 1990) is used to calculate the creep forces $f$ in the wheel-rail contact. However, the maximum creep force is limited to $f_{\text {max }}=F_{N} \mu$, with the normal wheel force $F_{N}$ and the friction coefficient $\mu$ between wheel and rail, see Figure 4. To further decrease the computational effort the creep torque is neglected (Polach, 2000) and the Kalker coefficients are kept constant. Thus, the contact formulation is stated as (Knothe and Stichel, 2003)

$$
\begin{gathered}
\boldsymbol{f}=\left(\begin{array}{c}
f_{x} \\
f_{y}
\end{array}\right)=\boldsymbol{K}\left(\begin{array}{c}
s_{x} \\
s_{y} \\
\phi_{z}
\end{array}\right), \text { with } \\
\boldsymbol{K}=-a b G\left(\begin{array}{ccc}
C_{11} & 0 & 0 \\
0 & C_{22} & \sqrt{a b} C_{23}
\end{array}\right) .
\end{gathered}
$$

The mathematical description of the nonlinear dynamics is carried out using three coordinate systems: the inertial (index $I$ ), the body fixed (index $\psi$, located in the middle of the axle bridge) and the contact point coordinate system (index $r, l$ ) (Jaschinski, 1990). However, the transformation matrices $\boldsymbol{A}$ converting these coordinate systems disregard the rotation of the wheels around the $y$-axis (Bremer, 1988).

The nonlinear model is deduced from the EulerLagrange equations

$$
\frac{\mathrm{d}}{\mathrm{d} t}\left(\frac{\partial T}{\partial \dot{\boldsymbol{q}}}\right)^{T}-\left(\frac{\partial T}{\partial \boldsymbol{q}}\right)^{T}+\left(\frac{\partial V}{\partial \boldsymbol{q}}\right)^{T}=\boldsymbol{Q}
$$

with the generalized forces $\boldsymbol{Q}$, the time derivative $\dot{\boldsymbol{q}}=$ $\left(\dot{y}_{f}, \dot{y}_{r}, \dot{\psi}_{f}, \dot{\psi}_{r}, \omega_{f r}, \omega_{f l}, \omega_{r r}, \omega_{r l}\right)^{T}$ of the generalized coordinates $\boldsymbol{q}$ and the kinetic and potential energy $T$ and $V$, respectively. With the masses of the frame $M$ and of an axle bridge including two wheels $m$, the moments of inertia of an axle bridge with respect to yawing $B$ and of a wheel with respect to rolling $C$ the kinetic energy $T$ results in

$$
\begin{aligned}
T= & \frac{M}{8}\left(\left(\sum \dot{y}_{i}\right)^{2}+\left(\sum \dot{z}_{i}\right)^{2}\right)+\frac{m}{2}\left(\sum \dot{y}_{i}^{2}+\sum \dot{z}_{i}^{2}\right)+ \\
& +\frac{B}{2}\left(\sum \dot{\psi}_{i}^{2}+\sum \dot{\alpha}_{i}^{2}\right)+\frac{C}{2}\left(\sum \omega_{i j}^{2}\right) .
\end{aligned}
$$

In addition, the potential energy $V$ is determined by two effects. The first is the potential $V_{s}$ of the rotational spring with the stiffness $k$ at the connection from the 
frame to the wheel carrier and the second is the elevation energy $V_{e}$

$$
V=V_{s}+V_{e}=\frac{k}{2} \psi_{f}^{2}+\frac{k}{2} \psi_{r}^{2}+(2 m+M) g z_{F} .
$$

In a next step, the velocities and displacements, needed for the calculation of the kinetic and potential energy, are determined. Since $y_{i}, \psi_{i}$ and $\omega_{i j}$ are generalized coordinates or time derivatives thereof, only $\alpha_{i}$ and $z_{i}$ have to be substituted for $\boldsymbol{q}$ and $\dot{\boldsymbol{q}}$, respectively. The kinematic relation between the rotation of the axle bridges about the $x$-axis and their translation along the $y$-axis is stated as (Jaschinski, 1990)

$$
\alpha_{i} \approx \tan \alpha_{i}=-\frac{d}{\frac{f}{2}-r_{0} d} y_{i}=-\Gamma y_{i}
$$

The vertical movements $z_{i}$ are on the one hand characterized through the yaw motions of the axle bridges and on the other hand through their lateral displacements. These influences can be treated separately (Jaschinski, 1990), so that the vertical displacements of the axle bridges are

$$
z_{i}=z_{i}\left(\psi_{i}\right)+z_{i}\left(\alpha_{i}\right)=\frac{d f}{2}\left(\frac{1}{\cos \psi_{i}}-1\right)+y_{i} \tan \alpha_{i} .
$$

The calculation of the generalized forces will be described through the right wheel of the leading axle bridge but can easily be transferred to the other wheels. The correlation between $\boldsymbol{Q}_{f r}$, the absolute and angular velocities of the wheel at the contact point and the creep force $\boldsymbol{f}_{f r}$ is

$$
\boldsymbol{Q}_{f r}=\left(\frac{\partial \boldsymbol{v}_{f r}}{\partial \dot{\boldsymbol{q}}}\right)^{T} \boldsymbol{f}_{f r}+\left(\frac{\partial \boldsymbol{\Omega}_{f r}}{\partial \dot{\boldsymbol{q}}}\right)^{T}\left(\begin{array}{c}
0 \\
\tau_{f} \\
0
\end{array}\right) .
$$

Thus, the required velocities are deduced as (Jaschinski, 1990)

$$
\begin{aligned}
& \boldsymbol{v}_{f r}=\boldsymbol{A}_{\psi I}\left(\begin{array}{c}
0 \\
\dot{y}_{f} \\
\dot{z}_{f}
\end{array}\right)+\left({ }_{\psi} \boldsymbol{\Omega}_{f r} \times\left(\begin{array}{c}
x_{f r} \\
y_{f r} \\
r_{f r}
\end{array}\right)\right), \\
& \text { with } \quad{ }_{\psi} \boldsymbol{\Omega}_{f r}=\left(\begin{array}{c}
0 \\
\omega_{f r} \\
0
\end{array}\right)+\left(\begin{array}{c}
\cos \psi_{f} \dot{\alpha}_{f} \\
-\sin \psi_{f} \dot{\alpha}_{f} \\
\dot{\psi}_{f}
\end{array}\right) .
\end{aligned}
$$

The actual rolling radius $r_{f r}$ is determined through the distance $y_{f r}$ from the axle bridge center to the contact point along the body fixed $y$-axis

$$
r_{f r}=\bar{r}-d \cdot y_{f r}, \quad \text { with } \quad \bar{r}=r_{0}+\frac{d f}{2} .
$$

Furthermore, $y_{f r}$ as well as the contact point shift $x_{f r}$ are dependent on the yaw angle and $y_{f r}$ is additionally dependent on the lateral displacement of the particular axle bridge.
At last, the creep force is calculated using (1), with the contact ellipse semi-axes $a$ and $b$ and the force $F_{N, f r}$ normal to the contact patch (Popp and Schiehlen, 2010)

$$
\sqrt{a b_{f r}}=\sqrt[3]{3 \frac{F_{N, f r}(1-\kappa) E_{g}}{2 \pi(A+D) G \sqrt{g_{v}}}}
$$

$A$ and $D$ are geometrical parameters determined by the curvature of the contacting bodies in the vicinity of the contact patch and therefore they vary with the yawing and the lateral displacement of the axle bridges. Nevertheless, $A$ and $D$ as well as the associated, dimensionless parameters $E_{g}$ and $g_{v}$ are set to a fixed value. Finally, the slip and spin of the nonlinear running gear model is

$$
\left(\begin{array}{c}
s_{x, f r} \\
s_{y, f r} \\
\phi_{z, f r}
\end{array}\right)=\frac{1}{v_{R}}\left(\begin{array}{c}
r v_{x, f r}+v_{R} \cos \psi_{f} \\
r v_{y, f r}-v_{R} \sin \psi_{f} \\
{ }_{r} \Omega_{z, f r}
\end{array}\right) .
$$

\subsection{Linear analytical Model}

On the basis of the model described in the previous section a linear analytical model in the form

$$
\boldsymbol{T} \dot{\boldsymbol{x}}=\boldsymbol{B} \boldsymbol{u}+\left(\boldsymbol{A}_{1}+\boldsymbol{A}_{2}\right) \boldsymbol{x}
$$

is generated, with $\boldsymbol{x}=\left(y_{i}, \psi_{i}, \dot{y}_{i}, \dot{\psi}_{i}, \Delta \omega_{i j}\right)^{T}$ and $\boldsymbol{u}=$ $\left(\tau_{f}, \tau_{r}\right)^{T}$. To receive the linear state space representation, $\Delta \omega_{i j}=\omega_{i j}-\omega_{0}$ is substituted for $\omega_{i j}$ (Goodall and Hong, 2000), with $\omega_{0}=-\frac{v_{R}}{r_{0}}$. Though the system gives no direct information about the angular wheel velocities, the accurately measurable velocity $v_{R}$ allows to recalculate $\omega_{i j}$.

The linearized equations of motion are presented in equation (13) and are discussed in the following. Due to the symmetry of the running gear the dynamics of the leading and the trailing axle bridge are determined in the same way. First of all, the differential equations of the lateral displacement show a coupling of the two axle bridges. In the scaled environment this mutual influence is quite small, since the mass of the axle bridge and the connected wheels $m$ is more than two times larger than the mass of the frame $M$. Regarding a 1:1 railway vehicle the mass ratio is vice versa and the coupling between front and rear axle bridge is more distinctive.

The part of the generalized forces referring to the torque $\tau_{i}$ constitutes the input matrix $\boldsymbol{B}$. The next term is the linearization of the derivative of the potential energy and represents the matrix $\boldsymbol{A}_{1}$. This vector shows clearly the two components of $V$, namely the elevation energy and the spring potential. In addition, the two influences on the elevation energy generated by the yaw and lateral motion, respectively, can be seen.

In terms of the linearization, the limitation of the creep forces at higher slip values illustrated in Figure 4 is repealed in this model. Nevertheless, the gap between the nonlinear and the linear creep force calculation can be 


$$
\begin{gathered}
\left(\begin{array}{c}
\left(\frac{M}{4}+m+B \Gamma^{2}\right) \ddot{y}_{f}+\frac{M}{4} \ddot{y}_{r} \\
\frac{M}{4} \ddot{y}_{f}+\left(\frac{M}{4}+m+B \Gamma^{2}\right) \ddot{y}_{r} \\
B \ddot{\psi}_{f} \\
B \ddot{\psi}_{r} \\
C \Delta \dot{\omega}_{f r} \\
C \Delta \dot{\omega}_{f l} \\
C \Delta \dot{\omega}_{r r} \\
C \Delta \dot{\omega}_{r l}
\end{array}\right)=\left(\begin{array}{c}
0 \\
0 \\
0 \\
0 \\
\tau_{f} \\
-\tau_{f} \\
\tau_{r} \\
-\tau_{r}
\end{array}\right)+\left(\begin{array}{c}
(2 m+M) g \Gamma y_{f} \\
(2 m+M) g \Gamma y_{r} \\
-\left[(2 m+M) \frac{g d f}{4}+k\right] \psi_{f} \\
-\left[(2 m+M) \frac{g d f}{4}+k\right] \psi_{r} \\
0 \\
0 \\
0 \\
0
\end{array}\right)+ \\
+\left(\begin{array}{c}
2 \bar{c}_{22} \eta\left(\psi_{f}-\frac{\zeta}{v_{R}} \dot{y}_{f}\right)-\frac{4 \bar{c}_{23} d}{f r_{0}}\left(\eta+\Gamma \frac{f d}{2}\right) y_{f}+\frac{\bar{c}_{23} \eta}{v_{R}}\left[2 \dot{\psi}_{f}+d\left(\Delta \omega_{f r}-\Delta \omega_{f l}\right)\right] \\
2 \bar{c}_{22} \eta\left(\psi_{r}-\frac{\zeta}{v_{R}} \dot{y}_{r}\right)-\frac{4 \bar{c}_{23} d}{f r_{0}}\left(\eta+\Gamma \frac{f d}{2}\right) y_{r}+\frac{\bar{c}_{23} \eta}{v_{R}}\left[2 \dot{\psi}_{r}+d\left(\Delta \omega_{r r}-\Delta \omega_{r l}\right)\right] \\
-\bar{c}_{11}\left[\frac{f d \zeta}{r_{0}} y_{f}+\frac{f r_{0}}{2 v_{R}}\left(\frac{f}{r_{0}} \dot{\psi}_{f}-\Delta \omega_{f r}+\Delta \omega_{f l}\right)\right]-\bar{c}_{23} f d\left(\frac{1}{r_{0}+r_{R}}-\frac{d^{2}}{r_{0}}\right) \psi_{f} \\
-\bar{c}_{11}\left[\frac{f d \zeta}{r_{0}} y_{r}+\frac{f r_{0}}{2 v_{R}}\left(\frac{f}{r_{0}} \dot{\psi}_{r}-\Delta \omega_{r r}+\Delta \omega_{r l}\right)\right]-\bar{c}_{23} f d\left(\frac{1}{r_{0}+r_{R}}-\frac{d^{2}}{r_{0}}\right) \psi_{r} \\
-\bar{c}_{11} r_{0}\left[-\frac{d \zeta}{r_{0}} y_{f}-\frac{f}{2 v_{R}} \dot{\psi}_{f}+\frac{r_{0}}{v_{R}} \Delta \omega_{f r}\right]-\frac{\bar{c}_{22} f d^{2}}{2\left(r_{0}+r_{R}\right)} \psi_{f} \\
-\bar{c}_{11} r_{0}\left[\frac{d \zeta}{r_{0}} y_{f}+\frac{f}{2 v_{R}} \dot{\psi}_{f}+\frac{r_{0}}{v_{R}} \Delta \omega_{f l}\right]+\frac{\bar{c}_{23} f d^{2}}{2\left(r_{0}+r_{R}\right)} \psi_{f} \\
-\bar{c}_{11} r_{0}\left[-\frac{d \zeta}{r_{0}} y_{r}-\frac{f}{2 v_{R}} \dot{\psi}_{r}+\frac{r_{0}}{v_{R}} \Delta \omega_{r r}\right]-\frac{\bar{c}_{23} f d^{2}}{2\left(r_{0}+r_{R}\right)} \psi_{r} \\
-\bar{c}_{11} r_{0}\left[\frac{d \zeta}{r_{0}} y_{r}+\frac{f}{2 v_{R}} \dot{\psi}_{r}+\frac{r_{0}}{v_{R}} \Delta \omega_{r l}\right]+\frac{\bar{c}_{23} f d^{2}}{2\left(r_{0}+r_{R}\right)} \psi_{r}
\end{array}\right) .
\end{gathered}
$$

kept within tolerable limits with the help of the actuator saturation. Hence, the generalized forces $\boldsymbol{Q}_{\boldsymbol{f}}$ caused by the creep forces determine the matrix $\boldsymbol{A}_{2}$. The vector in the second line of equation (13) describes this part of $\boldsymbol{A}$, using the dimensionless, geometrical parameters $\zeta=1+\Gamma r_{0}$ and $\eta=1+\Gamma \bar{r}$ and the extended Kalker coefficients $\bar{c}_{11}=a b G C_{11}, \bar{c}_{22}=a b G C_{22}$ and $\bar{c}_{23}=(a b)^{\frac{3}{2}} G C_{23}$. The parameters $\bar{c}_{11}$ and $\bar{c}_{22}$ have the dimension of a force and $\bar{c}_{23}$ the dimension of a torque. Considering the coefficients related to $\Delta \omega_{i j}$ it becomes obvious that the model is just linear in case of a constant roller velocity $v_{R}$. Furthermore, the linear model is only valid for this specific $v_{R}$, since the system behavior strongly depends on this parameter, what will be substantiated in the following section. Another aspect is that for a real configuration with longitudinal rails instead of rollers, the terms reciprocally proportional to $r_{R}$ vanish, because $r_{R} \rightarrow \infty$. Substituting common values for the parameters, it turns out that the influence of the spin is quite small in relation to the slip.

\section{Results}

After describing the three different models, they are compared in this section by the illustration of some substantial simulation results. At first, some analysis results of the linear model are presented to get more insight in the running gear dynamics. Figure 5 shows the eigenvalues of the linear model for $v_{R} \in\left[0.1 \frac{\mathrm{m}}{\mathrm{s}} ; 50 \frac{\mathrm{m}}{\mathrm{s}}\right]$ with steps of $0.1 \frac{\mathrm{m}}{\mathrm{s}}$. Considering the similarity laws stated in (Jaschinski, 1990) this velocity range corresponds to

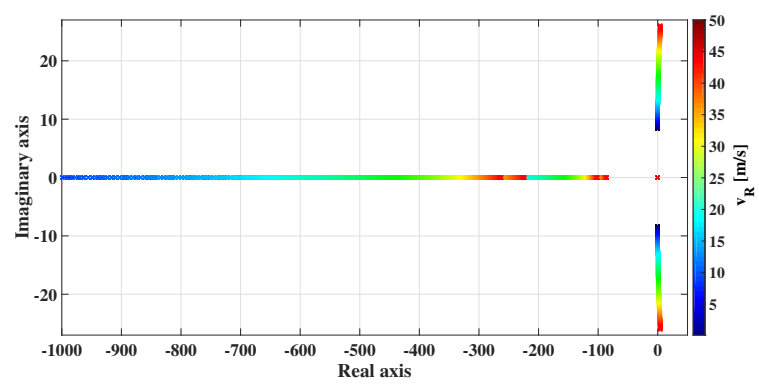

Figure 5. Eigenvalues of the linear model with varying roller speed $v_{R}$.

$\left[1 \frac{\mathrm{km}}{\mathrm{h}} ; 400 \frac{\mathrm{km}}{\mathrm{h}}\right]$ in a $1: 1$ configuration. According to equation (13), there are four first order terms, namely the equations describing the angular wheel motions, and four second order terms, namely the lateral and the yaw motions. Due to the symmetry of the running gear the eigenvalues of the front and the rear axle bridge occur in pairs. One of this eigenvalue pairs is at low velocities approximately $-1.3 \cdot 10^{5}$, since the coefficients reciprocally proportional to $v_{R}$ are very large in this case. In addition, it can be seen that with growing wheel velocity the real parts of all eigenvalues are moving in the positive direction, i. e. their dynamic behavior becomes slower. The detailed view of the area around the imaginary axis in Figure 6 shows that only two of the six eigenvalue pairs have got an imaginary part and consequently describe an oscillating behavior. This oscillating behavior called hunting motion is characteristic for railway vehicles with a conical or any nonlinear wheel profile. 


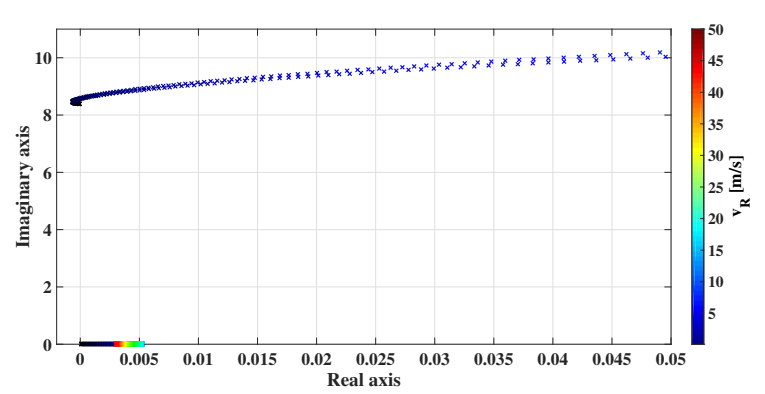

Figure 6. Unstable eigenvalues of the linear model with varying roller speed $v_{R}$.

Considering a conventional wheelset, the angular wheel velocities of the right and the left wheel are the same and in this way a self centering behavior occurs (Wickens, 2003). However, there is a critical velocity, that describes the maximum speed from which on the system is unstable. In (Dellmann and Abdelfattah, 2012) it was described that this hunting motion and the critical speed exist also for IRWs, what can be seen in Figure 6. In contrast to (Dellmann and Abdelfattah, 2012) the setup differs to some extent, e.g. the implementation of the yaw stiffness between the frame and the axle bridge. Because of this structural peculiarity, the eigenvalues describing the hunting motion possess already an imaginary part of about 8.4 at very low speeds. Another feature causing an instability is the potential energy, since a yaw motion of conical wheels comes along with a reduction of potential energy. This instability is characterized by the real eigenvalue in Figure 6.

In a next step, the transfer behavior from the input $u=\tau_{f}$ to the output $y=y_{f}$ of the real running gear is compared to the MBS model in Figure 7. The particular curves are created using a chirp signal as input (Saupe and Knoblach, 2012) and each is showing a nearly constant transfer behavior for frequencies of up to $1 \mathrm{~Hz}$. The green curve represents the running gear configuration without the redesign enhancements described in section 2. It shows an analog trend in relation to the measured roller rig data but has got a constant offset. The same resemblance characterizes the model that takes the newly constructed leaf spring guidance into account and

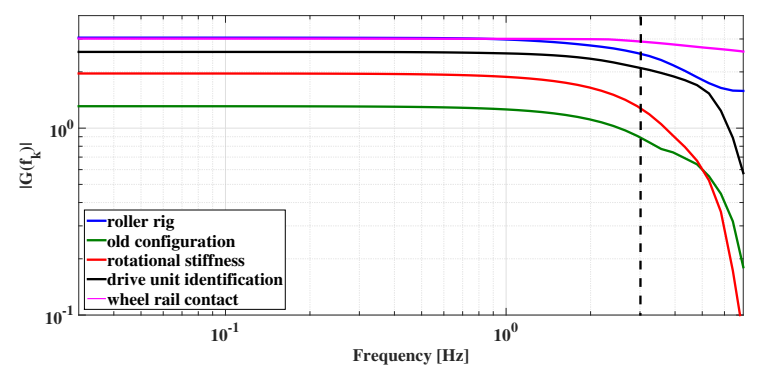

Figure 7. Measured and simulated transfer behavior of the running gear.
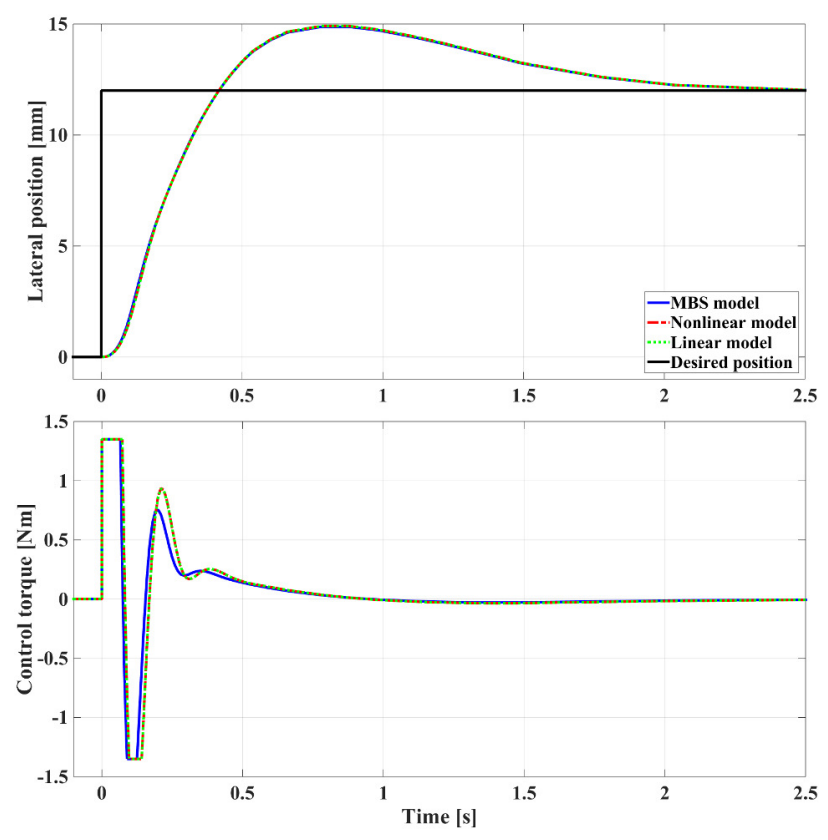

Figure 8. Desired and actual lateral displacement of the three simulation models at $v_{R}=2 \frac{\mathrm{m}}{\mathrm{s}}$.

the model with identified drive units in addition. Finally, the MBS model that additionally has got adapted wheel/rail contact parameters, e. g. coefficient of friction, conforms the hardware running gear very well in the area up to $3 \mathrm{~Hz}$. This adjustment shifts the drop, that can be seen for the other designs between 4 and $6 \mathrm{~Hz}$, to higher frequencies. Nevertheless, another parameteridentification will be done to further match the dynamic behavior of the simulation model also in the frequency range above $3 \mathrm{~Hz}$.

Since the detailed multibody model is validated with respect to the hardware running gear, the analytical models are compared to this model in the following two scenarios. Both simulations comprise a step of the desired lateral position so that the actuator torque reaches its limitation. Considering the applied roller speed the running gear with IRWs is an unstable system, so in consequence Figure 8 presents the results of the controlled running gear at a low velocity $v_{R}=2 \frac{\mathrm{m}}{\mathrm{s}}$. The lateral displacements of the three models in Figure 8 show a very good match and the corresponding results of the control torque verify the conformity of the three models. This means that congruent positions without just as congruent torques would not approve the model conformity at all. The control torques of the linear and the nonlinear model are nearly identical, so the nonlinearities in the running gear dynamics might be insignificant at least for the selected level of complexity in section 3.2.

Furthermore, a test scenario illustrated in Figure 9 is recorded at a three times higher wheel velocity than in Figure 8 . These simulation results show approximately the same distinguished conformity of the three models, though a slight deterioration at $t=0.2 \mathrm{~s}$ can be noticed 


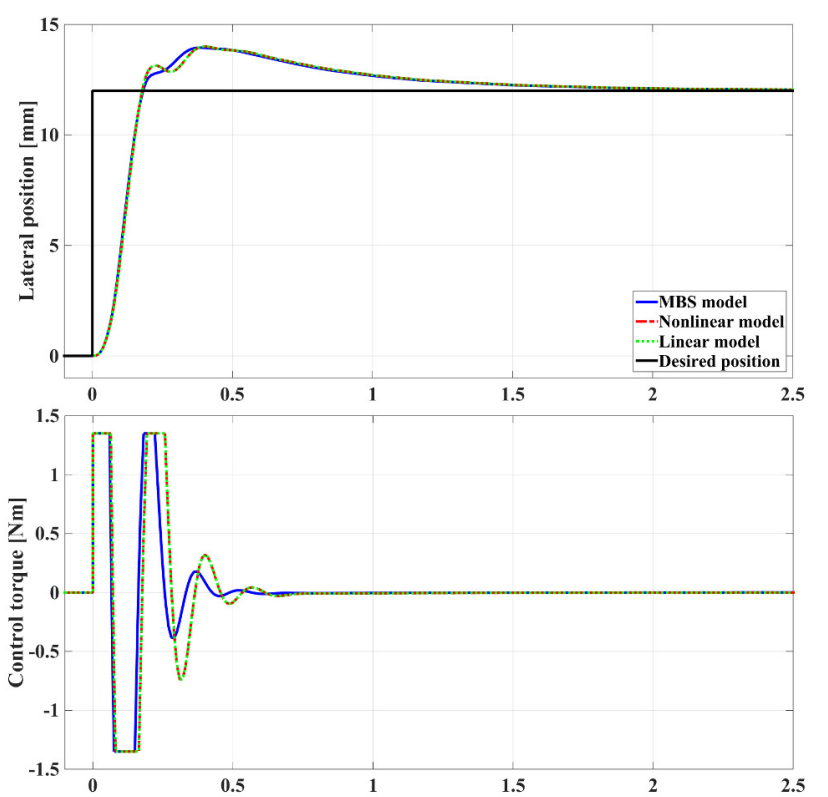

Figure 9. Desired and actual lateral displacement of the three simulation models at $v_{R}=6 \frac{\mathrm{m}}{\mathrm{s}}$.

because of the higher speed. This might be the outcome of the neglected DOFs, what is tolerated for the sake of a less complex model. Nevertheless, the results of the linear and the nonlinear model are as identical as in the low speed simulation affirming the validity of the linearization also for higher wheel velocities.

\section{Conclusions and Outlook}

Based on the scaled 1:5 running gear on the DLR roller rig three simulation models have been established. First of all, a detailed multibody model has been described that has been used and validated in former works at DLR. Furthermore, a nonlinear analytical model with a reduced complexity has been deduced in detail. The third implemented and tested running gear model is a linearization of the nonlinear analytical model. The comparison of the results of the three models approves their validity and enables their use in the development of advanced control and observer concepts. In this context, the analytical models will be inverted and used in a feedforward control concept (Heckmann et al., 2015). In addition, the excellent conformity of the linear and the nonlinear models facilitates the application of well-known linear analysis and control synthesis methods. Therefore, a plot of the eigenvalues has been illustrated as one of the results of the linear analysis of the running gear system and some characteristics of the dynamics of a railway vehicle with IRWs have been described.

Another aspect that can be scrutinized using the generated simulation models is a new sensor concept, since the laser sensors used for the measurement of $y_{i}$ would not accurately work in a real application due to dirt. Regarding this, force and torques sensors are already installed at the wheel mounting and shall after some further investigations replace the laser sensors. Finally, one part of the future work is to integrate the analytical models into the DLR RailwayDynamics Library to provide an environment for an advanced control development for railway systems.

\section{Acknowledgements}

This work was supported by BMBF (BMBF Förderkennzeichen: 01IS12022G), the German Federal Ministry of the Education and Research, within the ITEA 2 project Modrio.

\section{References}

H. Bremer. Dynamik und Regelung mechanischer Systeme. Teubner-Verlag, Stuttgart, 1988.

T. Dellmann and B. Abdelfattah. Comparison of dynamic properties of a conventional wheelset and an independently rotating wheelset - a theoretical contribution to an almost forgotten technology. ZEVrail, 136(10):380-390, 2012.

R. Goodall and L. Hong. Solid axle and independently-rotating railway wheelsets - a control engineering assessment of stability. Vehicle System Dynamics, 33(1):57-67, 2000.

A. Heckmann, A. Keck, I. Kaiser, and B. Kurzeck. The foundation of the DLR railwaydynamics library: the wheel-railcontact. In 10th International Modelica Conference, 2014.

A. Heckmann, C. Schwarz, T. Bünte, A. Keck, and J. Brembeck. Control development for the scaled experimental railway running gear of DLR. Vehicle System Dynamics, 2015. to appear.

A. Jaschinski. On the application of similarity laws to a scaled railway bogie model. Forschungsbericht DLR-FB 90-06, DLR, Institut für Dynamik der Flugsysteme, Oberpfaffenhofen, Germany, 1990.

J. Kalker. Three-dimensional elastic bodies in rolling contact, volume 2. Springer, 1990.

K. Knothe and S. Stichel. Schienenfahrzeugdynamik. Springer, Berlin, 2003.

O. Polach. A fast wheel-rail forces calculation computer code. Vehicle System Dynamics, 33:728-739, 2000.

K. Popp and W. Schiehlen. Ground Vehicle Dynamics. Springer, 2010.

F. Saupe and A. Knoblach. Design of excitation signals for the closed loop identification of industrial robots. In IEEE International Conference on Control Applications, 2012.

A. H. Wickens. Fundamentals of Rail Vehicle Dynamics: Guidance and Stability. Swets \& Zeitlinger, Lisse, NL, 2003.

J. Winter, E. Mittelbach, and J. Schykowski, editors. RTR Special - Next Generation Train. Eurailpress, DVV Media Group, 2011. 